\title{
Variación longitudinal de los elementos del leño en Schinopsis quebracho-colorado (Schlecht.) Barkl. et Meyer Anacardiaceae
}

\author{
Ana María Giménez ${ }^{1}$ \\ Carlos Raúl López ${ }^{1}$
}

\begin{abstract}
RESUMEN
El presente trabajo tiene como objetivo determinar el gradiente de variación longitudinal de los elementos anatómicos del xilema en Schinopsis quebracho-colorado (Schlecht.) Barkl. et Meyer, Anacardiaceae. Se trabajó con 10 individuos de DAP superior a $30 \mathrm{~cm}$, de la localidad Los Pirpintos, Departamento Copo, Santiago del Estero, Argentina. La metodología adoptada para el estudio del leño es la de Helinska et al. (1991) y la terminología de IAWA (IAWA, 1989). Las variables anatómicas seleccionadas son: espesor de anillos; longitud de vasos, de radios y de fibras; diámetro externo e interno de fibras; diámetro tangencial de vasos; número de vasos por milímetro cuadrado; y porcentaje de fibras, de parénquima y de vasos. El leño presenta un gradiente de variación longitudinal. Las variables anatómicas que determinan el gradiente en altura son: longitud de fibras, número de vasos por milímetro cuadrado y el diámetro interno de fibras. La longitud de fibras y el número de vasos por milímetro cuadrado, decrecen de la base al ápice. El diámetro interno de fibras se incrementa de la base al ápice. La mayor fuente de variabilidad es entre árboles. El leño presenta evidencias de baja heterogeneidad. Es necesario investigar con mayor profundidad el gradiente a alturas superiores a la estudiadas.
\end{abstract}

PALABRAS CLAVE:

Anatomía del leño, Schinopsis quebracho-colorado, variabilidad longitudinal.

\begin{abstract}
The aim of the study is to determine longitudinal gradient of variation of wood elements of Schinopsis quebracho-colorado (Schlecht.) Barkl. et Meyer, Anacardiaceae. The study was carried out in ten individuals from Los Pirpintos, Copo Department, Santiago del Estero, Argentina. Healthy, well formed trees, with more than $30 \mathrm{~cm} \mathrm{DBH}$ (diameter to the breast height, $1,30 \mathrm{~m}$ ) were selected from dominant canopy. Wood samples were taken following the methodology of Helinska et al. (1991) and terminology of IAWA (IAWA, 1989). Anatomical variable selects are: rings width, vessel number $/ \mathrm{mm}^{2}$, vessel tangential diameter, vessels and rays length, proportion of tissues, percentual type pore, external and internal diameter and fiber wall thickness. This wood presents a longitudinal gradient from base to apex. The characteristics that determine wood variability are: fibers length, number of pores $/ \mathrm{mm}^{2}$, and fibers wall thickness. Fiber length and number of pores $/ \mathrm{mm}^{2}$ decrese from base to apex. Fiber wall thickness increases from base to apex.The principal source of variability is between trees. Wood is heterogeneus. More investigations about heigt gradient are necessary.
\end{abstract}

KEY WORDS:

Wood anatomy, Schinopsis quebracho -colorado, longitudinal variability. 


\section{INTRODUCCIÓN}

La madera es una materia prima ampliamente usada en diferentes productos industriales. Actualmente, el mercado maderero exige calidad y uniformidad de productos. Las propiedades de la madera varían, debiéndose comprender la magnitud y las causas de estas variaciones (Zobel, 1984). La variabilidad de los elementos estructurales de la madera y sus propiedades físico-mecánicas reconocen diversas fuentes, que abarcan las variaciones originadas por razones geográficas, así como las variaciones entre y dentro de individuos.

Las principales variaciones de la madera se resumen en los siguientes patrones: variabilidad dentro del anillo de crecimiento, variabilidad producida de médula a corteza (variabilidad radial) y variabilidad ocasionada por diferentes niveles de altura (variabilidad longitudinal). Las propiedades del leño de numerosas especies varían de la base al ápice (Zobel y Van Buijtenen, 1989). Esta variación es frecuente en especies con grandes diferencias entre leño juvenil y maduro. El cambio de las propiedades en altura se produce automáticamente en función de la proporción de leño juvenil.

El efecto de la altura en las propiedades del leño no es constante en la madera de angiospermas. Briscoe et al. (1963) manifiestan que el patrón más frecuente en las maderas estudiadas es la escasa variabilidad en altura. Según Wilkens (1988) las variaciones en la anatomía del leño en sentido longitudinal son menos consistentes que las observadas en dirección radial.

En la actualidad, la bibliografía referente a la variabilidad de la madera en gimnospermas y en angiospermas es extensa, especialmente en los géneros Eucalyptus, Salix y Populus (Megraw, 1985). En angiospermas los antecedentes son menos numerosos, especialemente en el tema variabilidad longitudinal, destacándose los trabajos de Alipon
(1991), Kroll et al. (1992) y Land y Lee (1981).

En general, los estudios se centran en especies de zonas templadas, siendo muy limitados en latifoliadas tropicales y subtropicales. Entre ellos se enfatizan las investigaciones de Akachuku (1985) en Gmelina arborea; Wilkens (1988) en Eucalyptus sp.; León y Pernía (1999) en Cordia thaisiana; Giménez et al. (2001) en Populus deltoide, en las que se manifiesta que las variaciones anatómicas longitudinales son menos consistentes que las radiales.

En referencia a maderas nativas argentinas, son escasos los estudios de variabilidad del leño, citándose para la Región Chaqueña los trabajos de Giménez (1998, 2000), Giménez y López (2000), Moglia y López (2001a y 2001b) sobre las dos especies principales: el quebracho colorado y el quebracho blanco.

El objeto de investigar la variabilidad de la madera radica en la necesidad de materia prima homogénea para la industria. La variabilidad se agrava en los productos que provienen del bosque nativo donde la heterogeneidad genética de la población acentúa la disparidad del material.

El quebracho colorado santiagueño es la leñosa arbórea característica del Distrito Chaqueño Occidental y constituye el estrato superior (hasta $20 \mathrm{~m}$ de altura). Según la información consignada en el Inventario Forestal del Departamento Copo y Alberdi, Santiago del Estero, la especie es dominante en el bosque correspondiente al estrato I, siendo la de mayor volumen y posibilidad productiva (Thren y Zerda, 1994).

La madera es muy dura y pesada, con un peso específico aparente de 0,96 $\mathrm{kg} / \mathrm{dm}^{3}$ cuando tiene $0 \%$ de humedad y peso específico aparente de $1,03 \mathrm{~kg} / \mathrm{dm}^{3}$ cuando tiene $12 \%$ de humedad (Coronel, 1989). 
Una de las características más conspicuas es la presencia de sustancias tánicas en alta proporción en las distintas partes del vegetal. El contenido de fenoles totales en el leño es de $25 \%$, en la albura $6 \%$ y en el duramen $28,5 \%$ (Hillis, 1987). La corteza, hojas y frutos contienen taninos. Las estructuras de secreción constituyen un sistema interconectado en la planta.

Es muy utilizada como madera aserrada, pisos, postes, durmientes, combustible, producción de taninos. Este árbol orientó durante mucho tiempo la vida entera de la provincia de Santiago del Estero, constituyendo el pilar de su economía forestal (Cozzo, 1967). Se comercializan $50000 \mathrm{t}$ de madera para durmientes, leña, carbón, postes y madera rolliza. La heterogeneidad de la materia prima ha llevado a los investigadores de la zona a estudiar las posibles fuentes de variabilidad y sus causas.

\section{OBJETIVOS}

Explicar el gradiente de variación longitudinal de los elementos anatómicos del leño de Schinopsis quebrachocolorado (Schlecht.) Barkl. et Meyer, familia Anacardiaceae y analizar su fuente de variabilidad.

\section{METODOLOGÍA}

El estudio fue realizado en 10 individuos adultos de la especie Schinopsis quebracho-colorado (Schlecht.) Barkl. et Meyer, quebracho colorado santiagueño, perteneciente a la familia Anacardiaceae. Con el material de estudio se trabajó previamente en el tema crecimiento de árboles individuales y variabilidad radial del leño (Giménez, 1998).

El material se obtuvo del predio de la Cooperativa Forestal Los Pirpintos, de 5 000 ha, en la localidad de Los Pirpintos, Departamento Copo, Provincia de
Santiago del Estero, Argentina. Corresponde al Distrito Chaqueño Occidental y su posición geográfica es $25^{\circ} 58^{\prime} 28^{\prime \prime}$ Lat. S.; 62 09’04" Long. O.

Los individuos estudiados provienen del bosque nativo, fueron seleccionados según el criterio de: individuo adulto (diámetro a la altura del pecho, DAP superior a $30 \mathrm{~cm}$ ); con fuste sin defectos; árbol dominante; copa simétrica y sano. En él se midió el DAP, altura de fuste y altura total $y$, en el fuste, se marcó la dirección Norte.

Los árboles seleccionados fueron abatidos, se extrajeron rodajas de $5 \mathrm{~cm}$ de espesor, a las alturas de 1,30 m y 4,30 m (Helinska et al.,1991). Las muestras se trabajaron con cepilladora, lijadora de banda y orbital con juego de lijas de granulometría de 600 a 100, hasta tener una superficie lisa que demarcara con la mayor fidelidad los anillos de crecimiento.

Se trabajó con las muestras obtenidas a $1,30 \mathrm{~m}(\mathrm{H} 1)$ y a 4,3 m (H2) y el radio de orientación Norte. Se determinó una probeta de 10 anillos de crecimiento, de la posición central de la rodaja, correspondientes a la edad de 30 a 40 años. En una ventana de $2,5 \mathrm{~mm}$ de espesor, siguiendo la secuencia de los anillos marcados, se midieron los parámetros anatómicos año a año, observados en sección transversal y con lupa estereoscópica. Se estudiaron las siguientes variables anatómicas: espesor de anillo; longitud de vasos, de radio y de fibra; diámetro externo e interno de fibras; diámetro de vasos; vasos $/ \mathrm{mm}^{2}$; y proporción de tejidos del leño.

Las observaciones se efectuaron con lupa binocular (de la máquina cuenta anillos) y un aumento x 400 .

Para medir los caracteres anatómicos: alto y ancho de radios, diámetro externo e interno de fibras y espesor de pared, se efectuaron probetas correspondientes a los tratamientos $\mathrm{H}_{1}$ y $\mathrm{H}_{2}$, 
realizándose los respectivos cortes histológicos.

Las muestras, debidamente orientadas, se cortaron con micrótomo de carro móvil Leitz con un espesor de $12 \mu \mathrm{m}$ a $20 \mu \mathrm{m}$, en plano transversal y longitudinal tangencial y radial, teñidas con crisoidinaacridina roja y azul de astra. Posteriormente, se deshidrataron en serie alcohólica ascendente, se colocaron en xilol y se montaron con Entellán.

En las descripciones se siguió la terminología del Comité de Nomenclatura de IAWA (IAWA, 1989). Las mediciones anatómicas de los caracteres seleccionados se efectuaron siguiendo las recomendaciones de Muñiz y Coradin (1991), con la medición de 25 elementos por variable, individuo y tratamiento.

Se aplicó el análisis de la varianza (ANOVA) de Medidas Repetidas (Cody y Smith, 1991) para cada variable considerada, con el fin de estudiar su incidencia en la variabilidad total. El modelo aumenta la precisión a la prueba de $\mathrm{F}$ debido que considera al árbol como una variable clasificatoria y lo resta del error experimental. El objeto fue probar las posibles diferencias entre tratamientos a través de $\mathrm{F}$ y estimar los componentes de la varianza para la estimación de los parámetros en estudio según el modelo matemático siguiente:

$$
Y_{i j}=m+t_{i}+e_{i j}
$$

donde $m$ es la media aritmética evaluada en el tratamiento $i$ con $j$ individuos; $t$ es el efecto de los tratamientos; y $e_{i j}$ es el error experimental asociado a la parcela que contiene el tratamiento $i$. Se aplicó la Prueba de Duncan para conocer las diferencias entre tratamientos.

A fin de analizar las posibles fuentes de variabilidad de la madera en su gradiente longitudinal, se utilizó el método de máxima verosimilitud restringida
(REML). La participación porcentual de las variables clasificatorias sobre la variabilidad de los caracteres estudiados fue calculada por los componentes de varianza. Se usó el programa SAS v.6.12 para la realización del modelo

\section{RESULTADOS}

\section{Descripción anatómica}

El leño se caracteriza por presentar diferencia entre albura y duramen (Fig. 1a), con anillos de crecimiento moderadamente demarcados (Fig. 1b) y de contorno regular. La actividad cambial se inicia en el mes de septiembre y continúa hasta mayo (Giménez, 1998). El anillo está definido a nivel microscópico por una banda delgada de una a tres capas de fibras de paredes gruesas, tipo 3 de Coster (Fig. 1c).

La porosidad del leño es difusa no uniforme. Los poros son solitarios y múltiples de 2, 3 y 4, de orientación radial, con predominio de los poros solitarios en un $83 \%$.

Los miembros de vasos poseen placa de perforación simple. Son elementos cortos, de longitud media de 267,5 $\mu \mathrm{m}$ (200-500).

Los vasos son pequeños a medianos con un diámetro tangencial medio de 101,55 $\mu \mathrm{m}$ (40-200), numerosos a muy numerosos, con un valor medio de 9,54 vasos $/ \mathrm{mm}^{2}$ (8-35). El área media neta de vasos por $\mathrm{mm}^{2}$ es 0,0806 . Las puntuaciones intervasculares son alternas, coalescentes, con diámetro interno de puntuación $2 \mu \mathrm{m}$ a $5 \mu \mathrm{m}$, las parénquimavasculares $10 \mu \mathrm{m}$ a $16 \mu \mathrm{m}$ y las fibrovasculares $1 \mu \mathrm{m}$ a $2 \mu \mathrm{m}$ (Fig. 1d).

El índice de vulnerabilidad es 9,4 y el de mesomorfismo 2231,5 , correspondiendo a los valores de una especie mesofítica. 


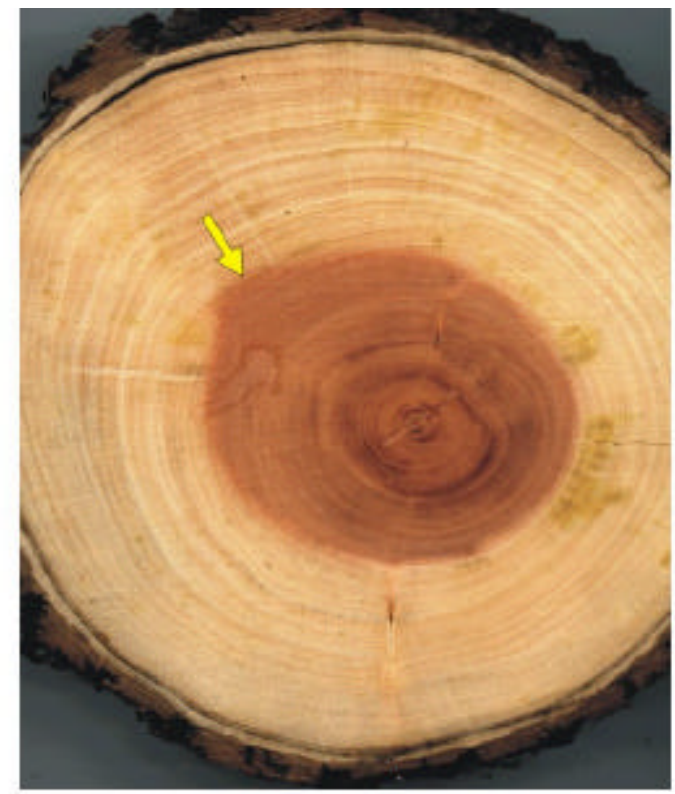

a
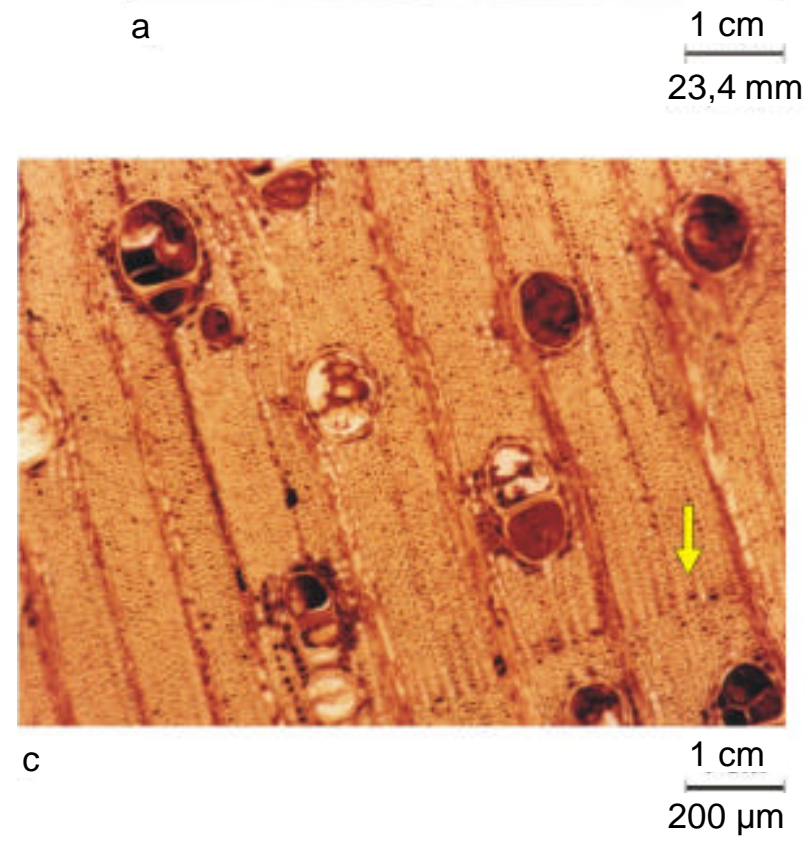

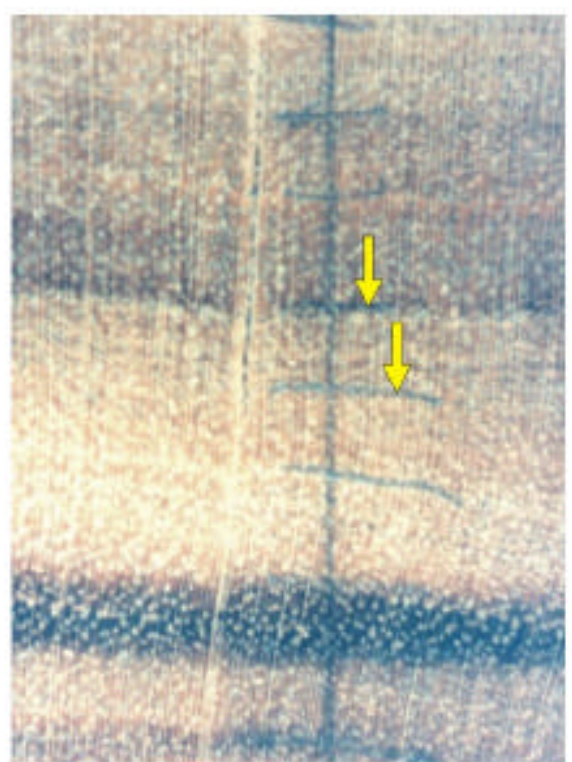

b

$$
\frac{1 \mathrm{~cm}}{4,86 \mathrm{~mm}}
$$

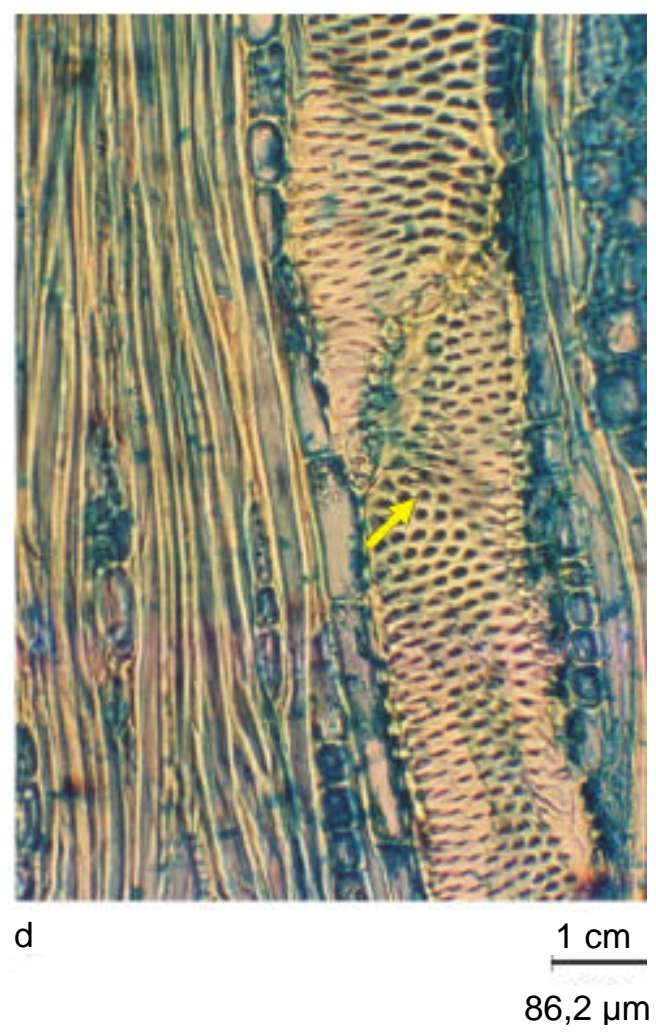

Figura 1. Leño de Schinopsis quebracho-colorado. a. Sección transversal del leño donde se evidencia albura y duramen; b. Anillos de crecimiento; c. Demarcación microscópica del anillo; $d$. Puntuaciones intervasculares. 
El tejido mecánico está constituido por fibras libriformes y fibrotraqueidas cortas, de longitud media de 904,90 $\mu \mathrm{m}$ (700 - 1 100). Las fibras son finas, de diámetro externo de un valor medio de $12,02 \mu \mathrm{m}$, diámetro interno de 6,62 $\mu \mathrm{m}$ y el espesor medio de pared de 2,97 $\mu \mathrm{m}$, correspondiendo al tipo grueso.

El parénquima axial es paratraqueal, vasicéntrico escaso. Los radios leñosos son heterogéneos II B Kribs, con células de contacto, multiseriados (4-3-2) y uniseriados. El rasgo anatómico sobresaliente es la presencia de canales secretores de taninos en los radios leñosos.

El leño presenta abundancia de cristales rómbicos de oxalato de calcio, dispuestos en el parénquima radial y en camadas de células septadas del parénquima axial.

Los vasos del duramen están ocluidos por tilosis, en algunos casos esclerosadas. El leño del duramen está totalmente impregnado por sustancias tánicas. Es frecuente observar en muestras tomadas durante el invierno la acumulación de abundantes gránulos de almidón en parénquima axial y fibras.

En la Tabla 1 se expresan los resultados estadísticos de los caracteres anatómicos del leño obtenido a partir de las muestras a las dos alturas estudiadas.

En la Tabla 2 se expresan los resultados del análisis de varianza según las variables clasificatorias árbol y altura.

Al no haber interacción arbol $x$ altura se continúa el tratamiento de los datos aplicando la Prueba de Duncan para las alturas $\mathrm{H} 1$ y $\mathrm{H} 2$ de las variables analizadas según se expresan en la Tabla 3.

Una vez explicadas las diferencias de las variables anatómicas con la altura mediante Duncan, se procede a analizar los componentes de la varianza. Las varianzas de las variables clasificatorias (árbol, altura) se estiman por REML para cada carácter anatómico según se indica en la Tabla 4. La representación porcentual de la variación asociada a cada fuente permite catalogar la incidencia de las mismas en la variabilidad del carácter en estudio.

La carencia de datos para longitud de vasos y porcentaje de parénquima se debe a que las variables clasificatorias árbol y altura no participan en los componentes de varianza. La variación está discriminada en el error experimental.

\section{DISCUSIÓN}

En primera instancia se considerará el gradiente longitudinal de los elementos axiales estudiados. La longitud de los elementos anatómicos posee un marcado efecto en la calidad de los productos y en el uso de las maderas, siendo este carácter importante en las coníferas (Webb, 1964).

En el quebracho colorado, ni la longitud de vasos ni la longitud de radios presentan un gradiente estadísticamente significativo. Esto coincide con León y de Pernía (1999) para Cordia thaisiana y Taylor (1977) para Celtis occidentalis.

La longitud de las fibras y miembros de vasos y el espesor de la pared celular influyen en el valor del producto final (Bosman et al., 1994). Por el contrario, el análisis de varianza del parámetro longitud de fibras presenta diferencias significativas para las variables clasificatorias árbol y altura. La Prueba de Duncan indica diferencias altamente significativas para las alturas, siendo superior en $\mathrm{H} 1$, por lo que se infiere que la longitud de fibras disminuye de base a ápice. 
Tabla 1. Estadística de las variables anatómicas del leño Schinopsis quebracho-colorado

\begin{tabular}{|c|c|c|c|c|c|}
\hline VARIABLE & MEDIA & $\begin{array}{l}\text { DESVIACIÓN } \\
\text { ESTANDAR }\end{array}$ & MÁXIMO & MÍNIMO & $\mathrm{CV}$ \\
\hline $\begin{array}{l}\text { Espesor de } \\
\text { anillos (mm) }\end{array}$ & 2,349 & 0,2642 & 2,909 & 1,947 & 41,31 \\
\hline $\begin{array}{l}\text { Longitud de } \\
\text { vasos }(\mu \mathrm{m})\end{array}$ & 267,40 & 47,49 & 350,00 & 150,00 & 17,28 \\
\hline $\begin{array}{l}\text { Longitud de } \\
\text { radios }(\mu \mathrm{m})\end{array}$ & 310,90 & 89,49 & 750,00 & 140,00 & 27,10 \\
\hline $\begin{array}{l}\text { Longitud de fibras } \\
(\mu \mathrm{m})\end{array}$ & 904,90 & 110,29 & 1100,00 & 600,00 & 10,89 \\
\hline $\begin{array}{l}\text { Diámetro externo } \\
\text { de fibras }(\mu \mathrm{m})\end{array}$ & 12,02 & 2,06 & 20,00 & 7,00 & 16,35 \\
\hline $\begin{array}{l}\text { Diámetro de } \\
\text { vasos }(\mu \mathrm{m})\end{array}$ & 101,55 & 25,91 & 180,00 & 40,00 & 23,3 \\
\hline Vasos $/ \mathrm{mm}^{2}$ & 9,54 & 3,96 & 19,00 & 3,00 & 21,53 \\
\hline $\begin{array}{l}\text { Diámetro interno } \\
\text { de fibras }(\mu \mathrm{m})\end{array}$ & 6,62 & 2,02 & 12,50 & 2,50 & 27,30 \\
\hline Fibras (\%) & 48,10 & 8,65 & 68,00 & 29,00 & 17,37 \\
\hline Parénquima (\%) & 24,88 & 6,97 & 37,00 & 10,00 & 31,49 \\
\hline Vasos (\%) & 26,78 & 7,57 & 46,00 & 11,00 & 26,04 \\
\hline
\end{tabular}

Tabla 2. Análisis de varianza (ANOVA) de los caracteres anatómicos para los tratamientos $\mathrm{H} 1$ y $\mathrm{H} 2$

\begin{tabular}{lccccc}
\hline $\begin{array}{l}\text { VARIABLE } \\
\text { ANATÓMICA }\end{array}$ & $\mathrm{F}$ & $\mathrm{Pr}>\mathrm{F}$ & ÁRBOL & ALTURA & ALT x REP \\
\hline Espesor de anillos & 1,23 & 0,6335 & 0,618 & 0,912 & 0,281 \\
\hline Longitud de vasos & 1,4 & 0,1001 & 0,5753 & 0,7150 & 0,036 \\
\hline Longitud de radios & 1,80 & $\mathbf{0 , 0 1 2 5}$ & $\mathbf{0 , 0 0 0 1}$ & $\mathbf{0 , 0 5 4 0}$ & 0,837 \\
\hline Longitud de fibras & 2,93 & $\mathbf{0 , 0 0 0 1}$ & $\mathbf{0 , 0 0 0 1}$ & $\mathbf{0 , 0 2 6 3}$ & 0,359 \\
\hline $\begin{array}{l}\text { Diámetro externo } \\
\text { de fibras }\end{array}$ & 1,7 & $\mathbf{0 , 0 2 1 5}$ & $\mathbf{0 , 0 0 5 2}$ & 0,0960 & 0,365 \\
\hline Diámetro de vasos & 2,42 & $\mathbf{0 , 0 0 0 3}$ & $\mathbf{0 , 0 0 0 1}$ & 0,9786 & 0,264 \\
\hline Vasos/mm & 20,27 & $\mathbf{0 , 0 0 0 1}$ & $\mathbf{0 , 0 0 0 1}$ & $\mathbf{0 , 0 0 0 1}$ & 0,831 \\
\hline $\begin{array}{l}\text { Diámetro interno } \\
\text { de fibras }\end{array}$ & 2,73 & $\mathbf{0 , 0 0 0 1}$ & $\mathbf{0 , 0 2 2 0}$ & $\mathbf{0 , 0 0 0 1}$ & 0,383 \\
\hline Fibras (\%) & 1,23 & 0,3110 & 0,2695 & 0,2190 & 0,5765 \\
\hline Parénquima (\%) & 0,32 & 0,9784 & 0,9229 & 0,6350 & 0,8981 \\
\hline Vasos (\%) & 1,58 & 0,1586 & 0,1356 & 0,3838 & 0,3499 \\
\hline "Las
\end{tabular}

* Las celdas en negrillas indican significancia al $95 \%$ de probalidad. 
Tabla 3. Prueba de Duncan para dos alturas

\begin{tabular}{|c|c|c|c|c|}
\hline VARIABLE & $\begin{array}{c}\text { GRUPO } \\
\text { DE } \\
\text { DUNCAN }\end{array}$ & MEDIA & $\begin{array}{l}\text { DESVIACIÓN } \\
\text { ESTANDAR }\end{array}$ & ALTURA \\
\hline \multirow[t]{2}{*}{ Espesor de anillos } & A & 2,3806 & 0,81 & $\mathrm{H} 1$ \\
\hline & A & 2,3206 & 0,85 & $\mathrm{H} 2$ \\
\hline \multirow[t]{2}{*}{ Longitud de vasos } & A & 269,0 & 46,27 & $\mathrm{H} 2$ \\
\hline & A & 265,8 & 50,79 & $\mathrm{H} 1$ \\
\hline \multirow[t]{2}{*}{ Longitud de radios } & A & 321,0 & 103,60 & $\mathrm{H} 1$ \\
\hline & A & 300,8 & 100,17 & $\mathrm{H} 2$ \\
\hline \multirow[t]{2}{*}{ Longitud de fibras } & A & 920,4 & 122,93 & H1 \\
\hline & B & 889,4 & 106,75 & $\mathrm{H} 2$ \\
\hline \multirow{2}{*}{$\begin{array}{l}\text { Diámetro externo de } \\
\text { fibras }\end{array}$} & A & 12,3 & 1,93 & $\mathrm{H} 2$ \\
\hline & $A$ & 11,8 & 2,24 & $\mathrm{H} 1$ \\
\hline \multirow[t]{2}{*}{ Diámetro de vasos } & A & 101,6 & 25,5 & $\mathrm{H} 1$ \\
\hline & A & 101,5 & 29,6 & $\mathrm{H} 2$ \\
\hline \multirow[t]{2}{*}{ Vasos $/ \mathrm{mm}^{2}$} & A & 12,79 & 3,07 & H1 \\
\hline & B & 6,29 & 4,63 & $\mathrm{H} 2$ \\
\hline \multirow[t]{2}{*}{ Diámetro interno de fibras } & A & 7,40 & 2,03 & $\mathrm{H} 2$ \\
\hline & B & 5,84 & 1,63 & H1 \\
\hline \multirow[t]{2}{*}{ Fibras (\%) } & A & 49,8 & 7,52 & $\mathrm{H} 1$ \\
\hline & A & 46,3 & 9,49 & $\mathrm{H} 2$ \\
\hline \multirow[t]{2}{*}{ Parénquima (\%) } & A & 25 & 6,43 & $\mathrm{H} 2$ \\
\hline & A & 25,5 & 7,36 & $\mathrm{H} 1$ \\
\hline \multirow{2}{*}{ Vasos (\%) } & A & 28,05 & 7,55 & $\mathrm{H} 2$ \\
\hline & A & 25,55 & 7,54 & $\mathrm{H} 1$ \\
\hline
\end{tabular}

El cambio de letras en los grupos expresa diferencias significativas para un nivel de probabilidad de 0,95 . 
Tabla 4. Varianza explicada por cada fuente de variación

\begin{tabular}{lcc}
\hline VARIABLE ANATÓMICA & \multicolumn{2}{c}{$\begin{array}{c}\text { COMPONENTE DE VARIANZA DE } \\
\text { VARIABLES CLASIFICATORIAS (\%) }\end{array}$} \\
\cline { 2 - 3 } & ÁRBOL & ALTURA \\
\hline Espesor anillo & 70 & 30 \\
\hline Longitud de vasos & - & - \\
\hline Longitud de radios & 88 & 12 \\
\hline Longitud de fibras & 87 & 13 \\
\hline Diámetro externo de fibras & 80 & 20 \\
\hline Diámetro de vasos & 100 & - \\
\hline Vasos/mm ${ }^{2}$ & 4,8 & 95,2 \\
\hline Diámetro interno de fibras & 15 & 85 \\
\hline Fibras (\%) & 70 & 30 \\
\hline Parénquima (\%) & - & - \\
\hline Vasos (\%) & 93 & 7 \\
\hline
\end{tabular}

Inokuma et al. (1956) observaron en Populus sp. el mismo gradiente que el indicado, mientras que Taylor (1977) describe un gradiente inverso en Celtis occidentalis. Para Liquidambar styraciflua (Webb, 1964) se cita la presencia de fibras más largas en la base, decreciendo con la altura del fuste. Gilmore (1971) indica que la madera más pesada está en la base del árbol y se aliviana en el ápice. Taylor y Wooten (1973) en Eucalyptus grandis indican que la longitud de fibras no está afectada por la altura, pero el diámetro de las células decrece.

La frecuencia de vasos $/ \mathrm{mm}^{2}$ es altamente significativa en el análisis de varianza para árbol y altura. $\mathrm{H} 1$ tiene mayor número de vasos $/ \mathrm{mm}^{2}$ que $\mathrm{H} 2$. El diámetro de vasos es una variable altamente significativa en el análisis de varianza para la variable clasificatoria árbol. No hay diferencias significativas para las alturas. Esto coincide con la tendencia citada por Rulliaty y America (1995) para Swietenia macrophylla. León y de Pernía (1999) indican diferencias significativas para el diámetro de vasos entre $3 \mathrm{~m}$ y $6 \mathrm{~m}$ de altura para luego mantenerse constante.
El diámetro externo de las fibras, no presenta diferencias significativas, siendo mayor en la altura H2. El diámetro interno de las fibras, presenta diferencias altamente significativas para $\mathrm{H} 1$ y $\mathrm{H} 2$, siendo mayor en H2. Calculado el espesor de la pared en función del diámetro externo e interno, $\mathrm{H} 1$ presenta pared de 3,03 $\mu \mathrm{m}$ y $\mathrm{H} 2$ de 2,45 $\mu \mathrm{m}$. Esta tendencia es similar a la analizada por Bosman (1996) en el primer tramo de altura.

La proporción de tejidos del leño no presenta diferencias significativas en altura, para ninguno de los parámetros, siendo las mismas que las determinadas por Giménez (1998) en el análisis del gradiente radial de la especie. La proporción de tejidos influye sustancialmente en el comportamiento y aptitud de la madera, variando con las especies (Rydholm, 1965).

El REML advierte que la variable árbol representa el $80 \%$ del componente de varianza en los caracteres: espesor de anillos, longitud de radios, longitud de fibras, diámetro externo de fibras, diámetro de vasos, porcentaje de fibras y porcentaje de vasos. 
Este gradiente de variación longitudinal entre árboles es el indicado por Bossman (1996), demostrando la importancia de una cuidadosa selección de las muestras para la investigación anatómica y las pruebas de calidad de madera.

En cambio, la variable clasificatoria altura representa un valor mayor del $80 \%$ para frecuencia de vasos $/ \mathrm{mm}^{2}$ y diámetro interno de fibras. Ello nos permite decir que sólo dos de las variables estudiadas explican el gradiente de variabilidad longitudinal del leño. Por lo anteriormente expuesto, se evidencia que no sólo hay que investigar en la variabilidad del leño sino su causa.

A diferencia de lo que ocurre con el gradiente de variación radial de la madera, se pueden encontrar los más variados patrones de variación longitudinal tanto en coníferas como en dicotiledóneas, por lo tanto hay que investigarlo en cada una de la especies de interés comercial.

\section{CONCLUSIONES}

- Schinopsis quebracho-colorado presenta un gradiente de variación longitudinal de los elementos anatómicos del leño determinado por las variables: longitud de fibra, numero de vasos $/ \mathrm{mm}^{2}$ y el diámetro interno de fibras.

- Para las dos primeras variables el gradiente longitudinal decrece de base a ápice siendo a la inversa para el diámetro interno de las fibras.

- La mayor fuente de variación del leño es producida por diferencias entre árboles, por lo que se concluye que el leño presenta evidencias de baja heterogeneidad.

- Es necesario investigar con mayor profundidad el gradiente a alturas superiores a la estudiadas.

\section{REFERENCIAS}

Akachuku, A. 1985. The effect of some extrinsic an intrinsic factors on the proportion of vessels in Gmelina arborea. Wood Science and Technology 19:1-12.

Alipon, M. 1991. Relative density and shrinkage of yemane (Gmelina arborea Roxb.) at different age and height. FPRDI J. 20(3/4):50-60.

Bosman, M. 1996. Longitudinal variation in selected wood properties of naturally and plantation grown light red meranti (Shorea leprosula, $S$. parvifolia, $S$. pauciflor, Dipterocarpaceae). IAWA 17(1):5-15.

Bosman, M.; I. Kort; M. van Genderen y P. Baas. 1994. Radial variation in wood properties of natural and plantation grown light red meranti (Shorea, Dipterocarpaceae). IAWA J.15:111120.

Briscoe, C.; J. Harris y D. Wyckoff. 1963. Variaton of specific gravity in plantation grown trees of bigleaf mahogany. Carib. For. 24:67-74.

Cody, R. y J. Smith. 1991. Applied statistic and the SAS. Programming language. North Holland. 3a. ed. Nueva York, Amsterdan, Londres. $404 \mathrm{p}$.

Coronel, E. 1989. Estudio y determinación de las propiedades físico-mecánicas de las maderas del parque chaqueño. 1a. parte. Instituto de Tecnología de la Madera. Facultad de Ciencias Forestales. Serie de publicaciones 8906. $28 \mathrm{p}$.

Cozzo, D. 1967. La Argentina Forestal. Editorial Universitaria de Buenos Aires. 240 p.

Giménez, A. 1998. Influencia de la edad en caracteres anatómicos del leño de 
Schinopsis quebracho-colorado, Anacardiaceae. Tesis Doctoral. FAZ. UNT. 110 p.

Giménez, A. 2000. Gradiente radial de los elementos anatómicos del leño en Schinopsis quebracho-colorado. Bosque 21(2):37-45.

Giménez, A. y C. López. 2000. Caracteres anatómicos que determinan la variabiliadad del leño de Schinopsis quebracho-colorado, Anacardiaceae. Revista de Investigaciones Agrarias(INIA) Sistemas y Recursos Forestales. España. Vol. 9(2):219-235.

Giménez, A.; R. Mellace y C. López. 2001. Variabilidad longitudinal y radial del leño de Populus deltoide cv. Catfish 5. En prensa.

Gilmore, A. 1971. Specific gravity of plantation grown yellow poplar is not related to site. Wood Fiber 13:182183.

Helinska, R.; E. Raczkowska y G. Fabisiak. 1991. Radial variation and growth rate in the length of the axial elements of sessile oak wood. IAWA Bull. 12(3):257-262.

Hillis, W. 1987. Heartwood and tree exudates. Springer Series and Wood Science. p:267

IAWA. 1989. IAWA List of Microscopy features for Hardwood Identification.

Inokuma, T.; K. Shimaji y T. Hamaya. 1956. Mesuarement of fibre lenght and specific gravity of Japanese gigant poplar (Populus japono-gigas). Misc. Infor-Tokio. Univ. For. 11:77-86.

Kroll, R.; D. Ritter y R. Gertjejansen. 1992. Anatomical and physical properties of balsam poplar (Populus balsamifera L.) in Minnesota. Wood fiber Sci. 4:13-24.
Land, S. y J. Lee. 1981. Variation in sycamore wood specific gravity in the South. Wood Sci. 13:166-170.

León, W. y N. de Pernía. 1999. Variabilidad de la madera de Cordia thaisiana Agostini (Boraginaceae) en sentido longitudinal. Revista Forestal Venezolana 43(1):33-42.

Megraw, R. 1985. Wood quality factors in loblolly pine. TAPPI Press. Atlanta, Georgia. 89 p.

Moglia, J. y C. López. 2001a. Variabilidad radial de algunos caracteres anatómicos de Aspidosperma quebrachoblanco. Bosque 22(2):3-14.

Moglia, J. y C. López. 2001b. Tendencia de variación radial del leño en Aspidosperma quebracho-blanco. Revista de Investigaciones Agrarias (INIA) Sistemas y Recursos Forestales. España. Vol. 10(1):69-80.

Muñiz Bolzón, G. y V. Coradin. 1991. Norma de procedimientos en estudios de anatomía da madeira. II Gimnospermae Comissae de estudios. CE: 11.01.07.002. ABNT. Brasilia: Laboratorio de Produtos Florestais, Serie Técnica.

Rulliaty, S y W. America.1995. Natural variation in wood quality indicators of Indonesian Big-Leaf Mahogany (Swietenia macrophylla). http://www. metla.fi/conf./iufro95abs/d5pos9. hmt.

Rydholm, S. 1965. Pulping processes. Interscience, Nueva York. 1269 p.

Taylor, F. 1977. Variation in specific gravity and fiber length on selected hardwood throughout the Mid South. For. Sci. (23):190-194.

Taylor, F. y T. Wooten. 1973. Wood property variation of Mississippi delta hardwoods. Wood and Fiber 5:2-13. 
Thren, M. y H. Zerda. 1994. Inventario Forestal de la Provincia de Santiago del Estero. Departamentos Copo y Alberdi. Convenio GTZ - UNSE - CFI. $114 \mathrm{p}$.

Webb, C. 1964. Natural variation in specific gravity, fiber length, and interlocked grain of sweetgum (Liqidambar styraciflua) in the South Atlantic States. Ph.D Tesis School for North California State Univ. Raleigh, Carolina del Norte. 125 p.
Wilkens, J. 1988. Variation in wood anatomy within species of Eucalyptus sp. IAWA Bull.n.s. 9:13-23.

Zobel, B. 1984. The changing quality of the world wood supply. Wood Sci. Technol 18:11-117.

Zobel, B. y J. Van Buijtenen. 1989. Wood variation. Its causes and control. Springer Series in Wood Science. Springer, Verlag. 355p.»

1 Instituto de Silvicultura y Manejo de Bosques (INSIMA). Facultad de Ciencias Forestales. Universidad Nacional de Santiago del Estero. Avda. Belgrano 1912 (s). 4200 Santiago del Estero, Argentina. c.e.: amig@unse.edu.ar.

Manuscrito recibido el 7 de febrero de 2002.

Aceptado el 30 de abril de 2002.

Este documento se debe citar como:

Giménez, A.M. y C.R. López. 2002. Variación longitudinal de los elementos del leño en Schinopsis quebracho-colorado (Schlecht.) Barkl. et Meyer Anacardiaceae. Madera y Bosques 8(2):27-38. 